\title{
A multimodal analytical method to simultaneously determine monoacetyldiacylglycerols, medium and long chain triglycerides in biological samples during routine lipidomics
}

\section{Raymond Thomas ( $\sim$ rthomas@grenfell.mun.ca )}

Memorial University of Newfoundland https://orcid.org/0000-0002-3078-2352

Charles Manful

Memorial University of Newfoundland

Thu Pham

Memorial University of Newfoundland

\section{Research Article}

Keywords: Thin layer chromatography, triglyceride analysis, acylated triglyceride, liquid chromatography, mass spectrometry, functional lipids, Eurosta solidaginis

Posted Date: July 16th, 2021

DOl: https://doi.org/10.21203/rs.3.rs-713915/v1

License: (c) (1) This work is licensed under a Creative Commons Attribution 4.0 International License.

Read Full License 
A multimodal analytical method to simultaneously determine monoacetyldiacylglycerols, medium and long chain triglycerides in biological samples during routine lipidomics

$10{ }^{1}$ School of Science and the Environment/ Boreal Ecosystem Research Initiative, Grenfell

11 Campus, Memorial University of Newfoundland, Corner Brook, NL A2H 5G4, Canada

14 *Corresponding author:

15 rthomas@grenfell.mun.ca || phone: +1 7096377161

16 cfmanful@mun.ca || phone: +1 4142079431

17

18

19

20

21

22 


\section{Abstract}

Introduction: Monoacetyldiglycerides (MAcDG), are acetylated triglycerides (TG) and an emerging class of bioactive or functional lipid with promising nutritional, medical, and industrial applications. A major challenge exists when analyzing MAcDG from other subclasses of TG in biological matrices, limiting knowledge on their applications and 33 metabolism.

34 Objective and Methods: Here in we demonstrate a multimodal analytical method for resolution, identification and quantitation of MAcDG in biological samples based on thin layer chromatography-flame ionization detection complimentary with C30-reversed phase liquid chromatography-high resolution accurate mass tandem mass spectrometry. We further apply this method to determine the MAcDG molecular species composition and quantity in E. solidaginis larvae.

Results and Conclusion: These findings suggest that the proposed analytical method could simultaneously provide a fast, accurate, sensitive, high throughput analysis of MAcDG from other TG subclasses, including the fatty acids, isomers and molecular species composition. We believe this method will allow for MAcDG to be included during routine lipidomics analysis of biological samples and will have broad interests and applications in the scientific community in areas such as nutrition, climate change, medicine and biofuel innovations.

Keywords: Thin layer chromatography, triglyceride analysis, acylated triglyceride, liquid 


\section{1. Introduction}

64 Triglycerides (TG) are neutral lipids which provide primary energy reserves in living 65 organisms ${ }^{1}$. Structurally, TGs are triesters composed of three fatty acid (FA) esterified at 66 the stereospecifically numbered carbon 1 (sn-1), 2 (sn-2), and 3 (sn-3) positions of the

67 glycerol backbone. They are involved in regulation of fatty acid metabolism and plasma 68 levels of lipoproteins in human ${ }^{2,3}$. TGs are divided into four subclasses based on their 69 fatty acids. In this regard, monoacetyldiglycerides (MAcDG) are acetylated triglycerides 70 having an acetate moiety $\left(\mathrm{CH}_{3} \mathrm{COO}-\right)$ at $s n-3$ position and longer fatty acyl chains at $s n-1$

71 and sn-2 positions. Short chain triglycerides (scTG) contain at least one fatty acyl chain 72 composed of $3-5$ carbons. Medium chain triglycerides (mcTG) contain at least one fatty 73 acyl chain composed of $6-12$ carbons, while long chain triglycerides (IcTG) are 74 composed of fatty acyl chains containing more than 12 carbon atoms ${ }^{4}$. IcTG are dietary 75 sources of essential polyunsaturated fatty acids including docosahexaenoic acid (DHA-

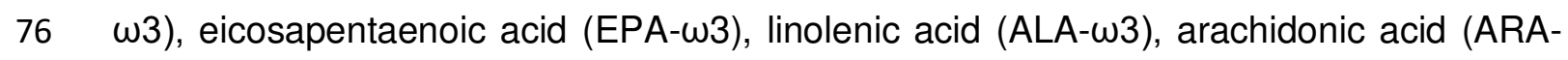
$77 \omega 6)$, and linoleic acid (LA-w6). Several nutritional studies support consumption of these 78 fatty acids and a reduction in risk factors for cardiovascular diseases, cancers, arthritis, 79 diabetes, hypertension and neuropsychiatric disorders ${ }^{5}$. Furthermore, consumption of 80 mcTG either in the diet or as supplements has been reported to be beneficial for reducing 81 body fat and obesity ${ }^{6}$, alcoholic liver diseases ${ }^{7}$, and short bowel syndrome ${ }^{8}$. MAcDG

82 have been reported to be effective in treating sepsis ${ }^{9,10}$, inflammations, cancers ${ }^{11}$, arthritis $83{ }^{12}$, and asthma ${ }^{13}$. Furthermore, MAcDG is an emerging functional lipid in the dietary and 
health sector. It is also a useful adjuvant for improving the performance of biofuels under 85 cold climatic conditions ${ }^{14,15}$.

86 As such, to fully exploit these benefits from MAcDG, there is a need for fast, accurate, 87 precise and sensitive techniques to identify and quantify MAcDG from other TG 88 subclasses in biological matrices. A major challenge exists in the scientific community with 89 analyzing MAcDG from other subclasses of TG. In most routine lipidomics techniques 90 used, MAcDG is not resolved from the other subclasses of TG, and as such there is limited 91 publications concerning their applications in the scientific community. Furthermore, there

92 is current interest in the biofuel industry to use MAcDG as an adjuvant to resolve viscosity 93 issues with biofuels when used in cold or northern climates. As a result, oil seed crops 94 such as camelina is now successfully engineered to produce high levels of MAcDG for 95 biofuel production and applications in cold climates ${ }^{14,15}$. Thus, there is a need for the development of suitable analytical methods to permit the routine analysis of MAcDG to

97 improve our understanding of how this unique lipid function and resolve issues in health and industrial applications. The literature is replete with analytical methods for the analysis of TG in biological samples that could be useful for the analysis of MAcDG ${ }^{16}$. Among 100 these, thin layer chromatography coupled with flame ionization detection (TLC-FID) 101 provides cost effective, accurate, precise, and efficient method for routine quantification 102 of TG subclass composition ${ }^{17-19}$. However, TLC-FID does not provide information on 103 molecular species and fatty acid compositions, which is important for nutritional, medical, 104 and industrial applications of TG. While mass spectrometry-based techniques including 105 gas chromatography coupled to flame ionization detection (GC-FID) and gas 106 chromatography coupled to mass spectrometry (GC-MS) provide higher sensitivity, 107 accuracy, precision, linear range and throughput compared to TLC-FID for quantification 108 of TG fatty acid composition, these methods are not applicable for quantification of intact 109 molecular species composition of TG subclasses including MAcDG ${ }^{19,20}$. Furthermore, 
$110 \mathrm{acid} / \mathrm{base}$ hydrolysis and chemical derivatization steps required for sample preparation in

111 GC-FID and GC-MS analysis of TG limits the general utility and scope of biological

112 samples which can be analyzed by these methods ${ }^{21}$. Liquid chromatography-heated 113 electrospray ionization tandem mass spectrometry (LC-HESI-MS/MS) allows identification 114 and quantitation of intact TG molecular species, fatty acid, and subclass composition of 115 biological samples in a single run ${ }^{22,23}$. Furthermore, LC-HESI-MS provides higher 116 accuracy, precision, and sensitivity compared to GC-MS and GC-FID, and does not 117 require prior hydrolysis and derivatization steps for mass spectrometric analysis of TG ${ }^{24}$. 118 We herein describe a multimodal analytical method for routine identification and 119 quantitation of MAcDG as a subclass of TG in biological samples based on TLC-FID 120 complimentary with C30-reversed phase liquid chromatography-tandem mass 121 spectrometry (C30-RPLC-MS/MS). We further applied this method for quantification of the 122 molecular species, and fatty acid composition in biological samples using $E$. solidaginis 123 larvae fat as an example. To the best of our knowledge, the proposed C30-RPLC-MS/MS 124 method is the first to be applied for quantification of MAcDG molecular species, fatty acid 125 and subclass compositions in E. solidaginis larvae fat. Furthermore, the proposed TLC126 FID method supplements our previous work 20 and demonstrates resolution of 127 hydroxylated MAcDG (OH-MAcDG) in relation to MAcDG, mcTG, and IcTG by TLC-FID. 128 We demonstrate the applicability of the proposed method for the analysis of MacDG during 129 routine lipidomics assessments of biological samples.

\section{2.0. Materials and methods}

131 2.1. Standards and reagents

132 Triglyceride (TG) standard mix (Splash Lipidomix Standard- 17811-1AMP) was obtained 133 from Supelco (Oakville Ontario, Canada). Synthesized and purified MAcDGs compounds, 134 including 16:0/18:0/2:0, 16:0/9Z-18:1/2:0,16:0/9Z,12Z-18:2/2:0 and OH-MacDG 
16:0/18:0(OH)/2:0, were provided by Chemforce Laboratories (Edmonton, Canada).

136 Calibration standard solutions $(0-10 \mu \mathrm{g} / \mathrm{mL})$ containing these standards were used as

137 spiked internal standards and to prepare standard curves to quantify TG in E. solidaginis.

\section{2.2. Collection of E. solidaginis larvae}

139 E. solidaginis larvae were extracted from the galls of Solidago spp. plants into $2 \mathrm{~mL}$ 140 Eppendorf centrifuge tubes and stored at $-80^{\circ} \mathrm{C}{ }^{20}$. These plants were from old field 141 habitats in London, Ontario, Canada $\left(43^{\circ} 00^{\prime} \mathrm{N}, 81^{\circ} 15^{\prime} \mathrm{W}\right)$. Memorial University Animal 142 Care provided ethics approval [20160041] for this study. All experiments complied with 143 established guidelines and regulations.

\subsection{Lipid extraction from $E$. solidaginis larvae}

145 Lipids were extracted in septuplicate $(\mathrm{N}=7)$ from $E$. solidaginis larvae $(85-170 \mathrm{mg})$ 146 according to the Folch method using $2.4 \mathrm{~mL}$ chloroform/methanol $(2: 1, \mathrm{v} / \mathrm{v})$ and $0.8 \mathrm{~mL}$ of $147 \quad 0.1 \% \mathrm{v} / \mathrm{v}$ butylated hydroxytoluene ${ }^{25}$. The resulting mixture was vortexed for 10 secs and 148 centrifuged at $10000 \times \mathrm{g}$ for $10 \mathrm{~min}$ at $-4^{\circ} \mathrm{C}$. The organic layers were pooled into pre149 weighed glass vials, and the solvent removed under dry nitrogen stream. Prior to TLC-FID 150 analysis, the dried lipids were dissolved in chloroform (700-900 $\mathrm{gg} / \mathrm{mL})$.

151 2.4. Thin layer chromatography-Flame ionization detection (TLC-FID) analysis of MAcDG 152 A standard mixture containing MAcDG, mcTG, IcTG and OH-MAcDG standards was used 153 for assessing the effectiveness of TLC-FID to resolve MAcDG from other classes of TG. 154 The TLC-FID analysis of TG subclass composition of the complex standard mixture was 155 conducted following the procedures described by Marshal et al. (2014) with minor 156 modifications ${ }^{20}$. Briefly, $1.7 \mathrm{~mL}$ solution of the complex standard mixture in chloroform 157 was resolved on chromarods ( $5 \mathrm{~mm}$ silica gel-coated quartz rod; Shell, Virginia, USA) 158 using 70:30:0.5 benzene:chloroform:formic acid (v/v/v) for chromatographic separation. 
TLC-FID analysis was performed using an latroscan thin-layer chromatograph (Model MK-

6 TLC-FID Analyzer; Shell, Virginia, USA) equipped with a flame ionization detector. The

161 instrument was operated at the following settings: flow rate of $2 \mathrm{~L} \mathrm{~min}^{-1}$ for atmospheric

162 air, $160 \mathrm{~mL} \mathrm{~min}^{-1}$ for hydrogen, and scanning speed of $3.0 \mathrm{~cm} \mathrm{~s}^{-1} 20$. Identification of TG

163 subclasses (MAcDG, mcTG, IcTG and OH-MAcDG) present in the complex standard 164 mixture was done by comparing retention times and elution order of the individual 165 standards (eg MAcDG) spotted on separate chromarods.

\subsection{MAcDG analysis by C30 reversed phase liquid chromatography-heated electrospray} ionization high resolution accurate mass tandem mass spectrometry (C30-RPLC-HESIHRAMS-MS/MS) and application for the analysis of MAcDG in E. solidaginis fat

169 In this section, we demonstrate the application of C30-RPLC-HRAMS-MS/MS for the 170 analysis of MAcDG in a complex standard mixture and a biological sample (E. solidaginis 171 fat) containing several different subclasses of TG (MAcDG, mcTG, ScTG, IcTG). LC-MS 172 analysis of $E$. solidaginis fat followed the same procedures described in our previous 173 publications ${ }^{26,27}$. Briefly, an Accucore C30 column (150 × 2 mm I.D., particle size: $2.6 \mu \mathrm{m}$, 174 pore diameter: $150 \AA$ А; Thermo Fisher Scientific, ON, Canada) coupled to a Dionex 175 Ultimate 3000 ultra-high performance liquid chromatography (UHPLC) system and a Q176 Exactive Orbitrap high resolution accurate mass spectrometer (Thermo Fisher Scientific, 177 ON, Canada) was used to resolve the lipids present in the complex standard mixture and 178 E. solidaginis larvae fat. For chromatographic separation, solvent A composed of 179 acetonitrile:water (60:40 v/v) while solvent B contained isopropanol:acetonitrile:water $180 \quad(90: 10: 1 \mathrm{v} / \mathrm{v} / \mathrm{v})$; with both solvents containing $10 \mathrm{mM}$ ammonium formate and $0.1 \%$ formic 181 acid. TG concentrations in E. solidaginis larvae fat are expressed on nanomol percent 182 (nmol \%) and microgram per gram fresh weight ( $\mu \mathrm{g} / \mathrm{g} \mathrm{FW})$ basis. 
185 Xcalibur data acquisition and interpretation software

186 (https://www.thermofisher.com/order/catalog/product/OPTON-30965\#, version 4.0,

187 Thermo Fisher Scientific, Ontario, Canada) was used for the C30-RPLC-HRAM-MS/MS

188 lipidomics data acquisition and processing. For the identification at a molecular level, the 189 relative positions of medium and long-chain fatty acids (FA) in TG molecular species

190 were assigned based on the general rules of the relative abundances of fragment ions in 191 their MS/MS spectra. The rule was established based on common findings that the loss 192 of fatty $\operatorname{acid}(\mathrm{s})$ at the sn-1 and sn-3 positions are observed at higher abundance than sn1932 fatty acid loss ${ }^{28-30}$. However, it was found to be an exception for TG containing short 194 chain fatty acids. For example, in this study we observed that the loss of $\mathrm{CH}_{3} \mathrm{COONH}_{4}$ 195 from the MS/MS spectra of MAcDG standards was at the lowest abundance although 196 acetic acid was esterified to the sn-3 position. For relative quantitation, integrated peak 197 areas and exact masses of TG molecular species were used to calculate the relative 198 abundance (nmol \%) of TG molecular species in E. solidaginis larvae fat. Furthermore, 199 the absolute quantitation was conducted based on a limited number of available 200 standards to estimate the concentration of TG molecular species in E. solidaginis larvae 201 fat $(\mu \mathrm{g} / \mathrm{g} F W)$. It was based on integrated peak areas of TG molecular species and 202 standards curves generated from authenticated standards (Triglycerides mixtures203 17811-1AMP) purchased from Supelco (Oakville Ontario, Canada) and MAcDG 204 standards obtained from Chemforce Laboratories (Edmonton, Canada). The standard 205 curves were generated using representative standards from each class due to the lack 206 of standards for all individual molecular species, and thus not completely accounted for 207 the matrix effects common in biological samples. However, it was found that the 208 corresponding factor of lipid ions depended mainly on their headgroup, thus only one 
209 representative standard is required for each lipid class/subclass in absolute quantitation

$210 \quad 31,32$.

211 2.7. Statistical analysis

212 TG molecular species and subclass composition in E. solidaginis larvae fat were assessed

213 using the descriptive statistics procedure in XLSTATS. One-way analysis of variance

214 (ANOVA) was used to determine significant differences between the levels of TG 215 molecular species and subclasses comprising the distributions of TG present in $E$. 216 solidaginis larvae fat. Where differences were significant, the means were compared with

217 Fisher's Least Significant Difference (LSD), $\alpha=0.05$. XLSTAT (Premium version, 218 Addinsoft, New York, USA) was used for statistical analysis.

\section{3.0. Results and discussion}

221 3.1. Analysis of MAcDG in complex lipid standard mixture using thin layer chromatography

222 coupled to flame ionization detection (TLC-FID)

223 One objective of this study was to demonstrate thin layer chromatography coupled with 224 flame ionization detection (TLC-FID) as a simple analytical method for routine, accurate 225 identification and quantitation of MAcDG from other TG subclasses in complex biological 226 samples. In the TLC-FID approach, we utilized a solvent system composed of 227 benzene:choloform:formic acid optimized for chromatographic separation of neutral lipid 228 classes on polar silica chromarods ${ }^{20,33}$. We observed that TG subclasses in the complex 229 standard mixture separated into four major peaks based on retention times corresponding 230 to OH-MAcDG (0.41 min), MAcDG (0.25 min), mcTG (0.22 $\mathrm{min})$, and IcTG (0.18 $\mathrm{min})$ 231 subclasses (Fig. 1a). The retention times demonstrated the polarity of the different TG 232 subclasses using this solvent system is as follows: $O H-M A c D G<M A c D G<m c T G<$ IcTG 
233 (Fig. 1a). The resolution of MAcDG and IcTG subclass are consistent with that reported 234 by Marshall at al. (2014) for the analysis of $E$. solidaginis larvae fat ${ }^{20}$. The concentration 235 range observed to provide excellent resolution and quantitation using TLC-FID is 100 $2361000 \mu \mathrm{g} / \mathrm{mL}(0.1$ to1 $\mathrm{mg} / \mathrm{mL})$. Below $100 \mu \mathrm{g} / \mathrm{mL}$ the peak size and appearance is not 237 consistent and above $1000 \mu \mathrm{g} / \mathrm{mL}$ we observed very large, broad shaped peaks that can 238 interfere with the resolution of MAcDG from the different TG subclasses. Furthermore, the 239 response is linear between $100-1000 \mu \mathrm{g} / \mathrm{mL}$ for the quantification of MAcDG as well as 240 other TG subclasses. The amount of MAcDG in a sample can be quantified by TLC-FID 241 from the average peak area of MAcDG (7 chromarods) present in each sample using the 242 linear regression equation $(y=m x+c)$ and calculated as follows ${ }^{17}$ :

$$
\operatorname{MAcDG}(\mu \mathrm{g} / \mathrm{g} \text { sample })=\frac{(y-c) \times V}{m \times W}
$$

244 where $y=$ average peak area of MAcDG, $c$ and $m=$ intercept and slope of the regression 245 line, respectively, $V=$ total volume of lipid solution $(\mathrm{mL})$, and $W=$ weight of sample used $246(g)$.

247 The output presented in this paper demonstrate the ability of the method for simple, rapid, 248 and sensitive quantification of OH-MAcDG, MAcDG, mcTG and IcTG in biological 249 samples. To the best of our knowledge, this is the first time hydroxylated MAcDG $(\mathrm{OH}-$ 250 MAcDG) has been resolved from other lipid classes using TLC-FID. This presents 251 opportunities for purification and further evaluation of the hydroxylated version of MAcDG. 252 which is a more polar version of MAcDG, as well as determine possible functions and 253 applications. It is well recognised that the bioactivities and physical properties of MAcDG 254 is distinctly different from that of other TG subclasses. How hydroxylation further 255 influences these properties is unknown. The application of TLC-FID to resolve and 256 quantify polar and neutral lipid classes in complex biological samples ranging from animal 257 (egg yolk, chicken fat, lamb fat, milk), marine (lobster, krill oil, red porgy wild, greater 
258

weever, piper gurnard) plant (sesame seeds, canola gum, macadamia nuts, olive oil), and edible fungus (mushrooms) origins have been widely reported ${ }^{19,34-36}$. These applications demonstrate the versatility of this analytical technique for routine, simple, efficient, accurate and sensitive analysis of neutral lipids. MAcDG can now be included as a subclass in routine neutral lipid analysis in these sources using TLC-FID. The proposed TLC-FID method provides a simple, comparatively inexpensive, sensitive and rapid method to separate, identify and quantitate TG subclasses classes including MAcDG and $\mathrm{OH}-\mathrm{MAcDG}$ in biological samples. In particular, the unique biochemical composition of MAcDG in biological samples including $E$. solidaginis can confer novel uses and application in the food science field (considering insects are emerging sources of dietary proteins and health-promoting functional lipids), healthcare (treating sepsis, cancers and tumors), and biofuel industry (potential additive to improve performance of biofuels in cold climates ${ }^{26,37}$. These uses suggest possible applications for MAcDG for which rapid, relatively inexpensive, accurate and sensitive analytical methods for quantification will be essential. Our findings demonstrate that TLC-FID could be used to accomplish this analysis across a range of biological samples.

\subsection{Analysis of MAcDG in complex lipid standard mixture using C30 reversed phase liquid} chromatography coupled to heated electrospray ionization- high resolution accurate mass tandem mass spectrometry (C30-RPLC-HESI-HRAM- MS/MS)

We further demonstrated that C30-reversed phase liquid chromatography coupled to high resolution accurate mass tandem mass spectrometry (C30-RPLC-HRAM-MS/MS) could be used complimentary to TLC-FID for the simultaneous analysis of the fatty acids and molecular species composition of intact MAcDG and other TG subclasses in biological samples. To this end, we used a mobile phase consisting of solvent A (acetonitrile: $\mathrm{H}_{2} \mathrm{O}$ $60: 40 \mathrm{v} / \mathrm{v}$ ) and solvent B (isopropanol:acetonitrile:water 90:10:1 v/v/v) both containing 
ammonium formate buffer as the solvent system applied with C30-RPLC coupled with 284 accurate mass tandem mass spectrometry optimized for separation and quantification of 285 intact neutral lipid species ${ }^{26}$. We observed clear resolution of TG molecular species 286 including mcTG, IcTG MAcDG, and OH-MAcDG present in standard mixture based on 287 polarity, fatty acid composition and fatty acid chain length ranging from $\mathrm{TG}(8: 0)_{3}$ to $288 \mathrm{TG}(18: 1)_{3}$, in positive ion mode (Fig. 1b). Resolution of non-acetylated TG species 289 including mcTG: TG(8:0) $)_{3}$ occurred at $16.47 \mathrm{~min}$, $\mathrm{TG}(10: 0)_{3}$ at 20:00 min and $\mathrm{TG}(12: 0)_{3}$ 290 at $22: 39$ min from IcTG: $\mathrm{TG}(14: 0)_{3}$ at $24: 88 \mathrm{~min}, \mathrm{TG}(16: 1)_{3}$ at $25: 14 \mathrm{~min}, \mathrm{TG}(16: 0)_{3}$ at $29127.95 \mathrm{~min}$ and $\mathrm{TG}(18: 1)_{3}$ at $28: 27 \mathrm{~min}$ standards was achieved. These non-acylated TG 292 molecular species were well separated from the acetylated TG including OH-MAcDG 293 which occurred at 19:77 $\mathrm{min}$ and MAcDG at 21:53 min (Fig. 1b). The presence of $\mathrm{OH}$ 294 group in OH-MAcDG appears to have increased the relative polarity of MAcDG-OH 295 thereby reducing its retention on the C30-RP column relative to MAcDG and IcTG species 296 in the standard mixture ${ }^{38}$. As such MAcDG-OH eluted before MAcDG and IcTG species, 297 as shown in Fig. 1b. Furthermore, separation of mcTG and IcTG molecular species 298 present in the standard mixture was based on their fatty acid chain lengths ${ }^{26}$. It is accepted 299 that hydrophobic interactions between the stationary phase and hydrophobicity of fatty 300 acyl chains (based on chain length, number and position of double bonds of fatty acids) 301 provides the resolution of neutral lipid species present in the standard mixture ${ }^{39}$. McTG 302 molecular species have shorter fatty acyl chains compared to IcTG species which makes 303 the former less hydrophobic compared to IcTG. As such, mcTG molecular species were 304 less retained under C30-RPLC conditions compared to the more hydrophobic IcTG 305 species $^{40}$.

306 The high resolving power of the Q-Exactive Orbitrap mass spectrometer allowed for 307 accurate and unambiguous resolution and structural identification of TG molecular species 308 including MAcDG and OH-MAcDG present in the standard mixture in positive ion mode 
(Fig. 2). The C30-RPLC-HRAM-MS/MS spectra of MAcDG and OH-MAcDG molecular species present in the standard mixture are shown in Fig. 2a-d. Ammonium formate buffer was used in the C30-RPLC method for column optimization. Therefore, all intact TG

312 molecular species present in the standard mixture formed ammonium adducts $\left[\mathrm{TG}+\mathrm{NH}_{4}\right]^{+}$ 313 under the C30-RPLC-HRAMS-MS/MS conditions used in this study ${ }^{26,41}$. For example, the 314 MS/MS spectrum of MAcDG 16:0/18:1/2:0[M+NH$]^{+}$at $m / z 654.57$ is shown in Fig. 2a. 315 The diagnostic sn-3 acetyl moiety at $\mathrm{m} / \mathrm{z} 577.52$ corresponds to the neutral loss of $316\left[\mathrm{CH}_{3} \mathrm{COO}^{-} \mathrm{NH}_{4}{ }^{+}\right]$or $77 \mathrm{Da}$ from $\mathrm{m} / \mathrm{z} 654.57$ [MAcDG 16:0/18:1/2:0 $\left.+\mathrm{NH}_{4}\right]^{+}$molecular ion ${ }^{42}$. 317 The composition and positions of fatty acids (FA) in TG molecular species including 318 MAcDG and OH-MAcDG present in the standard mixture were identified based on the 319 neutral loss of the fatty acid fragments as ammonium adducts, and presence of the fatty 320 acid ketene ions $\left[F A+\mathrm{H}^{-} \mathrm{H}_{2} \mathrm{O}\right]^{+}$in the $\mathrm{MS} / \mathrm{MS}$ spectra ${ }^{26,43}$. As such for MAcDG $321 \quad 16: 0 / 18: 1 / 2: 0$ (Fig. 2a), the fragment at $m / z 355.28$ correspond to the neutral loss of $18: 1$ $322+\mathrm{NH}_{3}(s n-2 \mathrm{FA})$ or $299 \mathrm{Da}$, while the fragment at $\mathrm{m} / \mathrm{z} 381.30$ correspond to the neutral 323 loss of 16:0 $+\mathrm{NH}_{3}(s n-1 \mathrm{FA})$ or $273 \mathrm{Da}$ from $m / z$ 654.57 [MAcDG 16:0/18:1/2:0 $\left.+\mathrm{NH}_{4}\right]^{+}$ 324 ion ${ }^{44}$. Distinction between sn-2 and sn-1 fatty acids is based on the relative abundance of 325 these two FA fragments, with relative abundance of sn-2 FA fragment [18:1 $\left.+\mathrm{NH}_{3}\right]$ lower 326 than that of $s n-1$ FA fragment $\left[16: 0+\mathrm{NH}_{3}\right.$, which is a trend typical of $s n-1$ and $s n-2 \mathrm{FAs}$ 327 in $\mathrm{TG}^{45}$. Furthermore, fragment ions at $\mathrm{m} / \mathrm{z} 239.24$ and 265.25 in Fig. 2a were assigned 328 to fatty acid ketene ions $\left[16: 0+\mathrm{H}-\mathrm{H}_{2} \mathrm{O}\right]^{+}$and $\left[18: 1+\mathrm{H}-\mathrm{H}_{2} \mathrm{O}\right]^{+}$corresponding to sn-1 FA 329 and sn-2 FA, respectively. The distinction between sn-2 and $s n-1$ positions on TG 330 molecular species including MAcDG and OH-MAcDG could also be identified by the 331 relative abundance of these fatty acid ketene ions ${ }^{26}$. The relative abundance of $[18: 1+$ $\left.332 \mathrm{H}-\mathrm{H}_{2} \mathrm{O}\right]^{+}$ketene ion at $m / z 265.26$ corresponding to sn-2 FA was higher than that of [16:0 $\left.333+\mathrm{H}_{-} \mathrm{H}_{2} \mathrm{O}\right]^{+}$ion at $m / z 239.24$ for $s n-1 \mathrm{FA}$ of MAcDG 16:0/18:1/2:0, which is a trend typical 334 of sn-1 and sn-2 FA ketene ions in TG species (Fig. 2a). Thus, the trend based on relative 
abundance of $s n-1$ and $s n-2[F A+H-H 2 O]^{+}$ketene ions is opposite to that observed for

336 fragments formed by neutral loss of FAs from [MAcDG 16:0/18:1/2:0 $\left.+\mathrm{NH}_{4}\right]^{+}$ion, and is 337 consistent with the literature on determining TG molecular species ${ }^{43,44}$.

338 The proposed C30-RPLC-MS/MS method also allowed for resolution and identification of $339 \mathrm{OH}-\mathrm{MAcDG}$ molecular species in the complex standard mixture. Structurally, while $\mathrm{OH}-$ 340 MAcDG and MAcDG are distinguished from non-acetylated TG species including scTG, 341 mcTG and IcTG by the presence of sn-3 acetyl groups, OH-MAcDG differs from MAcDG 342 by having one hydroxyl group on the sn-2 fatty acid chain. These structural differences 343 were seen in the mass spectra of MAcDG and OH-MAcDG molecular species present in 344 the standard mixture under C30-RPLC-MS/MS conditions (Fig. 2a-b). For example, the 345 MS/MS spectra of OH-MAcDG 16:0/18:0(OH)/2:0 m/z 672.58 is shown in Fig. 2b. The 346 neutral loss of $\left[\mathrm{CH}_{3} \mathrm{COO}^{-} \mathrm{NH}_{4}^{+}\right]$or $77 \mathrm{Da}$ from $\left[\mathrm{TG}+\mathrm{NH}_{4}\right]^{+}$ions is representative of MAcDG 347 molecular species ${ }^{42}$. In contrast OH-MAcDG molecular species are distinguished from 348 MAcDG by the neutral loss of $\left[\mathrm{CH}_{3} \mathrm{COO}^{-} \mathrm{NH}_{4}{ }^{+}+\mathrm{H}_{2} \mathrm{O}\right]$ or $95 \mathrm{Da}$ from $\left[\mathrm{TG}+\mathrm{NH}_{4}\right]^{+}$ions ${ }^{46}$. 349 Accordingly, the fragment at $\mathrm{m} / \mathrm{z} 577.52$ corresponding to neutral loss of $\left[\mathrm{CH}_{3} \mathrm{COO}^{-} \mathrm{NH}_{4}{ }^{+}\right.$ $350+\mathrm{H}_{2} \mathrm{O}$ ] or $95 \mathrm{Da}$ from $\mathrm{m} / \mathrm{z} 672.58\left[\mathrm{OH}-\mathrm{MAcDG} \text { 16:0/18:0(OH)/2:0 }+\mathrm{NH}_{4}\right]^{+}$ion is diagnostic 351 of the sn-3 acetate moiety in OH-MAcDG 16:0/18:0(OH)/2:0 (Fig. 2b). Furthermore, 352 relative abundances of fatty acid fragments arising from neutral loss from $\left[\mathrm{TG}+\mathrm{NH}_{4}\right]^{+}$ions 353 and fatty acid ketene ions were used to assign sn-1 and sn-2 fatty acid composition of $354 \mathrm{OH}-\mathrm{MAcDG}$ species. Consistent with conventions in the literature for assigning the sn1 355 and sn2 fatty acids based on relative abundance ${ }^{45}$, fragments at $m / z 355.28$ (18:0 sn-2) 356 and $m / z 381.30(16: 0 \mathrm{sn}-1)$ correspond to neutral loss of $\left[18: 0+\mathrm{NH}_{3}\right]$ and $\left[16: 0+\mathrm{NH}_{3}\right]$ 357 from [OH-MAcDG 16:0/18:0(OH)/2:0 $\left.+\mathrm{NH}_{4}\right]^{+}$ion, respectively (Fig. 2b). As previously 358 alluded to, the relative abundance of fragment corresponding to sn-2 FA is lower 359 compared to sn-1 FA fragment ${ }^{45}$. In a similar fashion, the fragments at $m / z 239.24$ and $360 \mathrm{~m} / \mathrm{z} 265.25$ were diagnostic of fatty acid ketene ions $\left[16: 0+\mathrm{H}-\mathrm{H}_{2} \mathrm{O}\right]^{+}$and $\left[18: 0+\mathrm{H}-\mathrm{H}_{2} \mathrm{O}\right]^{+}$ 
respectively, with the relative abundance of the sn-2 FA ketene ion higher than the $s n-1$

362 FA ketene ion (Fig. 2b). In summary, neutral loss of $77 \mathrm{Da}$ and $95 \mathrm{Da}$ from $\left[\mathrm{TG}+\mathrm{NH}_{4}\right]^{+}$

363 ions was used to distinguish between MAcDG and OH-MAcDG molecular species present 364 in the standard mixture.

365 In contrast, non-acetylated TG including scTG, mcTG and IcTG present in the standard 366 mixture did not form fragments corresponding to neutral loss of $77 \mathrm{Da}$ and $95 \mathrm{Da}$ from [TG $\left.367+\mathrm{NH}_{4}\right]^{+}$ions under C30-RPLC-HRAM-MS/MS conditions, which provided a basis for their 368 identification relative to acylated TG (MAcDG and OH-MAcDG). For example, the MS/MS 369 spectra of mcTG 8:0/8:0/8:0 m/z 488.39 is shown in Fig. 2c. The neutral loss of $161 \mathrm{Da}$ is 370 representative of C8:0 FA loss from $\mathrm{m} / \mathrm{z}$ 488.39 $\left[\mathrm{mcTG} \text { 8:0/18:0/8:0 }+\mathrm{NH}_{4}\right]^{+}$ion, which 371 corresponds to the fragment at $m / z 327.25$ (Fig. 2c), while the fragment at $m / z 127.11$ 372 corresponds to the fatty acid ketene ion $\left[8: 0+\mathrm{H}-\mathrm{H}_{2} \mathrm{O}\right]^{+}$at $s n-1, s n-2$ and $s n-3$ positions of 373 mcTG 8:0/8:0/8:0 26. The same approach was applicable for structural elucidation of IcTG 374 molecular species present in the standard mixture. Accordingly, the $m / z 551.50$ fragment 375 in Fig. 2d corresponds to the neutral loss of FA 16:0 (273 Da) from $\mathrm{m} / \mathrm{z} 824.68$ [IcTG $\left.37616: 0 / 16: 0 / 16: 0+\mathrm{NH}_{4}\right]^{+}$ion, while fatty acid ketene ion $\left[16: 0+\mathrm{H}-\mathrm{H}_{2} \mathrm{O}\right]^{+}$was at $m / z 127.11$ 37726,28 . Application of C30-RPLC-MS/MS to identify and quantify neutral lipid molecular 378 species in biological samples have been reported in the literature. Narvaez et al. (2016) 379 applied a RPLC-MS/MS method in positive ion mode to resolve and analyze neutral lipids 380 including TG in rat liver and rat plasma lipid extracts ${ }^{22}$. A similar application has been 381 used for analysis of TG composition of Calu-3 cells, rat blood and human skin tissues ${ }^{47}$. 382 However, none of these techniques have included the analysis of MAcDG and $\mathrm{OH}$ 383 MacDG. We have developed the method utilizing a C30-RPLC-HRAM-MS/MS technique 384 to analyze neutral lipids including TG and was successful in detecting MAcDG in wild 385 cervid meats for the first time (moose and caribou) ${ }^{48}$. Taken together, the proposed C30386 RPLC-HRAM-MS/MS method allows for accurate detection and quantification of TG 
molecular species including $\mathrm{OH}-\mathrm{MAcDG}$ and MAcDG with good resolution, sensitivity, and

388 throughput during routine lipidomics.

\subsection{Application of C30-RPLC-HESI-HRAM-MS/MS for separation and identification of}

390 MAcDG molecular species in larvae of E. solidaginis

391 TG subclass and molecular species composition in E. solidaginis is of interest due to its

392 role as a model system in cold stress tolerance. In this model system, MAcDG have been

393 demonstrated to provide cryoprotection during exposure to low temperature stress ${ }^{20}$. This

394 work has also led to subsequent studies recently on the potential of producing specialized

395 oil seed crops with superior levels of MAcDG for applications in the production of biofuels

396 specific for cold temperatures or northern climates ${ }^{15,49}$. For example, Liu et al. (2015)

397 reported that EaDAcT genetically modified camelina and soybean accumulated MAcDG

398 at up to 70 mol\% of the total seed oil produced by the resultant crops. A similar strategy

399 of genetic modification increased MAcDG content to $85 \mathrm{~mol} \%$ in field-grown transgenic

400 camelina ${ }^{15}$. Furthermore, recent interests in insects as a potential source of proteins and

401 functional ingredients for food and animal feed to improve population health and wellbeing 402 has gained recognition, for which the larvae of $E$. solidaginis could be a promising food 403 source ${ }^{50,51}$. TG in E. solidaginis larvae fat have been reported to contain about 36 mol\% 404 MAcDG compared to long chain TG ${ }^{20}$. However, no report was done on the molecular 405 species composition of MAcDG in E. solidaginis. MAcDG is associated with several health 406 benefits in human and these include treating sepsis ${ }^{10}$, tumor growth and cancers ${ }^{11}$, 407 rheumatoid arthritis ${ }^{12}$ and asthma ${ }^{13}$.

408 We applied the proposed C30-RPLC-HRAM-MS/MS method to separate MAcDG lipid 409 species present in the fat of $E$. solidaginis larvae from other TG molecular species. We 410 observed MAcDG eluted between 18.9 to $20.64 \mathrm{~min}$ followed by the other TG subclasses 411 between 21.44 and 27.63 under C30-RPLC conditions (Fig. 3a-b). Four main classes of 
412 TG molecular species were identified in the larvae of $E$. solidaginis including MAcDG, 413 scTG, mcTG and IcTG (Fig. 3b). A clear separation of these TD subclasses was observed 414 based on hydrophobicity, chain lengths and molecular weight as follows: MAcDG< $\mathrm{scTG}<$ 415 mcTG $<$ IcTG (Fig. 3b). No OH-MAcDG specie was observed in E. solidaginis fat, which is 416 line with the literature ${ }^{20}$. The C30-RPLC separated the more polar MAcDG species from 417 non-acetylated TG species (scTG, mcTG and IcTG), which allowed for facile mass 418 spectrometric identification and quantification (Fig. 3b). High resolution accurate mass 419 tandem mass spectrometry (HRAM-MS/MS) was used to assign the molecular species 420 composition of MAcDG, scTG, mcTG and IcTG species in E. solidaginis larvae fat as 421 shown in Fig. 4a-e \& Fig. S-1 - S-3. For example, the MS/MS spectra of MAcDG $42218: 1 / 16: 1 / 2: 0[\mathrm{M}+\mathrm{H}]^{+}$at $m / z 652.56$ is shown in Fig. 4a. The fragment at $m / z 575.51$ 423 corresponds to the neutral loss of $77 \mathrm{Da}\left[\mathrm{CH}_{3} \mathrm{COO}^{-} \mathrm{NH}_{4}^{+}\right]$from $\mathrm{m} / \mathrm{z} 652.56$ [MAcDG $\left.42418: 1 / 16: 1 / 2: 0+\mathrm{NH}_{4}\right]^{+}$ion. This neutral loss $77 \mathrm{Da}$ is representative of the sn-3 acetyl 425 moiety $\left[\mathrm{CH}_{3} \mathrm{COO}-\mathrm{NH}_{4}{ }^{+}\right]$characteristic of all MAcDG species. The two fatty acid fragments 426 were at $m / z 381.31(16: 1 s n-2)$ and $m / z 353.27$ (18:1 sn-1) corresponding to neutral loss 427 of $\left[16: 1+\mathrm{NH}_{3}\right]$ and $\left[18: 1+\mathrm{NH}_{3}\right]$ from $\left[M A c D G \text { 18:1/16:1/2:0 }+\mathrm{NH}_{4}\right]^{+}$ion, respectively (Fig. 428 4a). Furthermore, corroboration of the fatty acid composition of MAcDG 18:1/16:1/2:0 was 429 also based on the diagnostic fatty acid ketene ions at $m / z 237.22$ (sn-2 FA) and $m / z 265.26$ 430 (sn-1 FA) which correspond to $\left[16: 1+\mathrm{H}-\mathrm{H}_{2} \mathrm{O}\right]^{+}$and $\left[18: 1+\mathrm{H}-\mathrm{H}_{2} \mathrm{O}\right]^{+}$ions, respectively (Fig. $4314 a)$. Assignment of sn-1 FA and sn-2 FA of MAcDG 18:1/16:1/2:0 was made based on the 432 relative abundance of the neutral loss FA fragments and fatty acid ketene ions as 433 elaborated previously in the discussion. A similar approach was applied to elucidate the 434 structures of other MAcDG species detected in E. solidaginis larvae fat (Fig. 4b \& Fig. S4351 - S-3). Significantly, the proposed C30-RPLC-HRAM-MS/MS method provided facile 436 resolution of MAcDG and TG molecular species present as isomers in E. solidaginis larvae 437 fat. For example, the MS/MS spectra of MAcDG species present in E. solidaginis larvae 
were distinguished from scTG, mcTG and IcTG species by the neutral loss of $\left[\mathrm{CH}_{3} \mathrm{COO}\right.$ $\left.\mathrm{NH}_{4}^{+}\right]$or $77 \mathrm{Da}$ from $\left[\mathrm{TG}+\mathrm{NH}_{4}\right]^{+}$ion, which is diagnostic of the sn-3 acetyl moiety of all MAcDG species (Fig. 4a-e). Using the approach based on the relative abundances of neutral loss of the fatty acid fragments and fatty acid ketene ions explained above, as well as in the literature ${ }^{52}$, the two isomers at $m / z 874.79\left[\mathrm{TG} 52: 3+\mathrm{NH}_{4}\right]^{+}$reported in Fig. $4 \mathrm{~d}$ e were assigned as IcTG 18:1/18:1/16:1 and IcTG 18:2/18:1/16:0 molecular species respectively.

Furthermore, it was possible to resolve isomers of MAcDG from that of other TG subclasses present in E. solidaginis larvae using the proposed method (Fig. 5a-b). For example, MAcDG 18:0/18:1/2:0 $[\mathrm{M}+\mathrm{H}]^{+} \mathrm{m} / \mathrm{z} 682.59$ eluted at 20.64 min under C30-RPLC conditions (Fig. 5b) is an isomer of scTG 16:0/18:0/4:0 [M+H] ${ }^{+} \mathrm{m} / \mathrm{z} 682.59$ which eluted at $21.44 \mathrm{~min}$ (Fig. 5a). These isomers were easily resolved based on their MS/MS fragmentation patterns (Fig. 5a-b). For ScTG 16:0/18:1/4:0, the three fatty acid composition were assigned as follows: the fragments at $\mathrm{m} / \mathrm{z} 577.53$ is diagnostic of $4: 0$ fatty acid at $s n-3, m / z 409.34$ for $16: 0$ at $s n-1$ and $m / z 383.32$ for $18: 1$ at the $s n-2$ positions of the glycerol moiety. This correspond to the neutral loss of $\left[4: 0+\mathrm{NH}_{3}\right],\left[16: 0+\mathrm{NH}_{3}\right]$ and $\left[18: 1+\mathrm{NH}_{3} \text { ] from } \mathrm{m} / \mathrm{z} 682.59 \text { [scTG 16:0/18:2/2:0 }+\mathrm{NH}_{4}\right]^{+}$ion, respectively (Fig. 5a). Assignment of the sn-1, sn-2 and sn-3 FAs of scTG 16:0/18:0/4:0 was based on the relative abundance of these fragments with sn-1 FA $>s n-2 \mathrm{FA}>s n-3 \mathrm{FA}$, a trend typical of TG species ${ }^{26,28}$. Furthermore, diagnostic fatty acid ketene ions at $\mathrm{m} / \mathrm{z} 239.24$ and $\mathrm{m} / \mathrm{z}$ 265.26 correspond to $\left[16: 0+\mathrm{H}-\mathrm{H}_{2} \mathrm{O}\right]^{+}$and $\left[18: 1+\mathrm{H}^{-} \mathrm{H}_{2} \mathrm{O}\right]^{+}$, which is representative of sn$1 \mathrm{FA}$ and sn-2 FA respectively (Fig. 5a). In contrast, the structure of MAcDG 18:0/18:1/2:0 $[\mathrm{M}+\mathrm{H}]^{+} \mathrm{m} / \mathrm{z} 682.59$ in Fig. 5b was distinguished from scTG 16:0/18:1/4:0 by the fragment at $m / z 605.56$ which correspond to neutral loss of $77 \mathrm{Da}$ from $\mathrm{m} / \mathrm{z} 682.59$ [MAcDG 18:0/18:1/2:0 $\left.+\mathrm{NH}_{4}\right]^{+}$ion. Assignment of the $s n-1$ and $s n-2 \mathrm{FAs}$ in MAcDG 18:0/18:1/2:0 was based on the relative abundance of the fragments associated with the neutral loss of 
each fatty acid and the fatty acid ketene ions (Fig. 5b). A similar approach was used to assign all TG and MAcDG molecular species including isomers present in $E$. solidaginis (Fig. 4-5 \& Fig. S1-S2). This work shows the advantage of C30-RPLC-HESI-HRAMMS/MS as a superior platform for resolving isomers of MAcDG from other TG subclasses in biological samples. We believe MAcDG maybe observed to be more common in biological samples if their analysis is facilitated during routine lipidomics.

The quantitation and distribution of MAcDG and other TG subclasses present in $E$. solidaginis larvae fat is shown in Fig. 6a-b. The fat of $E$. solidaginis is enriched with MAcDG accounting for $38 \%$ of the TG composition, while IcTG accounted for $48 \%$ on mass percent basis (Fig. 6c-d). These findings are consistent with those presented by Marshall et al. (2014) who demonstrated that the level of IcTG and MACDG in E. solidaginis was $36 \%$ and $29 \%$ respectively, and varied with seasons under cold temperature stress following analysis using TLC-FID ${ }^{20} \mathrm{~A}$ thorough discussion of the use of TLC-FID for analyzing MAcDG and long chain TG is presented in our previous publication ${ }^{20}$. We also demonstrated for the first time that the fat of $E$. solidaginis consisted of $12 \%$ and $5 \%$ scTG and mcTG respectively (Fig. 6c-d). We further identified a total of 34 TG molecular species in E. solidaginis, which consisted of 12 MAcDG species, 2 mcTG species, 2 scTG species and 17 ICTG species (Fig. 6e). The MAcDG molecular species composition was predominated by 18:1/18:1/2:0, while 18:1/18:1/4:0, 18:1/18:1/12:0 and 18:1/18:1/18:1 respectively predominated ScTG, mcTG, and IcTG compositions in E. solidaginis (Fig. 6e). Furthermore, the TG profile of E. solidaginis was enriched in PUFA TG consisting of 38 and 40 carbons, as well as two double bonds. Approximately, $48 \%$ of the TG species consisted of 2 double bonds compared to $71 \%$ for MAcDG (Fig. 6f-g). Furthermore, we observed that MADAG in E. solidaginis was composed of shorter carbon chains compared to TG (C34-C40 vs C34-C56) with C38 predominating in both. However, MADAG had higher levels of C38 carbon chains compared to other TG subclasses ( $69 \%$ vs $58 \%$ ) as is 
shown in Fig. 6f-g. The output from this method shows for the first time the molecular

491 species composition of MAcDG compared with other TG subclasses in E. solidaginis. This

492 is important considering E. solidaginis can survive at cold temperatures as low as $-80^{\circ} \mathrm{C}$

493 and is a well recognised model system for studying cold temperature stress in different

494 biological systems. MAcDG has been previously reported to confer cryoprotective 495 properties to $E$. solidaginis during temperature stress survival. Information on the 496 molecular species composition of MAcDG will allow further work by researchers in the 497 scientific community to improve our understanding of the mechanisms associated with 498 MAcDG metabolism during cold temperature stress acclimation. Furthermore, insect 499 larvae are an emerging source of high-quality dietary lipids. This information could be 500 useful in the evaluation of E. solidaginis as a source of dietary MAcDG or functional lipids. 501 In summary, the proposed C30-RPLC-HRAM-MS/MS method appears to be a suitable 502 approach for the analysis of MAcDG and other TG subclasses, molecular species, isomers 503 and fatty acid composition in E. solidaginis fat, that could also be applied for routine 504 lipidomics analysis of other biological samples.

505

506

507

508

\subsection{Advantages of a multimodal approach using high resolution C30-RPLC-HESI-HRAM-}

\section{MS/MS for routine MAcDG analysis.}

The multimodal approach proposed in this paper is advantageous compared to other approaches in the literature in that TLC-FID facilitate quick, cost effect simple resolution of MAcDG from other neutral lipids including subclasses of TG, (mcTG, scTG, IcTG), as well as polar lipids using silica rods and a non-polar solvent system. The flame ionization detection (FID) facilitates rapid, cost effective quantitation of total MAcDG in the sample. We used this approach to demonstrate for the first time the resolution of hydroxylated MAcDG from other TG subclasses. Applying C30-RPLC-HESI-HRAM-MS/MS allow determination of the molecular species composition including the fatty acids composition 
515 of MAcDG in biological samples. Most lipidomics analysis currently use C18 RPLC

516 columns to resolve TG and other neutral lipids during routine lipidomics. We use C30

517 RPLC instead, due to the superior resolving power of C30 stationary phase in separating

518 TG and other lipid isomers. When combined with accurate mass tandem mass

519 spectrometry this is a powerful platform to resolve MAcDG molecular species, fatty acids

520 and isomers including positional isomers as demonstrated in this paper. This is very

521 important because MAcDG is not typically analyzed as a component of routine or

522 untargeted lipidomics. Generally, a targeted approached is employed that often involved

523 pre concentration steps.

\section{4.0. Conclusion}

525 In this work, we present the utilization of TLC-FID and C30-RPLC-HESI-HRAM-MS/MS

526 as a multimodal approach for the analysis of MAcDG, OH-MAcDG, ScTG, mcTG and IcTG

527 during routine lipidomics analysis. TLC-FID separated TG lipids into subclasses based

528 on polarity, while C30 RPLC separated TG lipids based on chain length, polarity and fatty 529 acid composition. We successfully applied the C30-RPLC-HESI-HRAM-MS/MS method 530 to resolve MAcDG, scTG, mcTG and IcTG lipid subclasses, and molecular species and 531 isomers in E. solidaginis as an example of the suitability of the method for routine 532 lipidomics analysis of biological samples. Furthermore, C30-RPLC-HESI-HRAM-MS/MS 533 provided excellent intra-class resolution of TG subclasses and isomers with different fatty 534 acid compositions. We believe this method will allow for MAcDG to be included in routine 535 lipidomics of biological samples and will have broad interests and applications in the 536 scientific community.

\section{Author contribution}



research; T.H.P., C.F.M and H.S. performed lab analyses; T.H.P. and C.F.M performed

540 formal data analysis. C.F.M prepared manuscript draft. R.H.T., C.F.M. and T.H.P. 541 reviewed the manuscript. All authors contributed to editing the final version of manuscript.

542 Competing interests

543 The authors declare no competing interests.

544 Acknowledgement: We thank Memorial University of Newfoundland and NSERC for 545 funding this study. We thank Dr. Yuan Tao for maintaining instruments used in our 546 analysis. We are very grateful to Dr. B.J. Sinclair of University of Western Ontario for 547 providing spherical galls of Solidago spp. plants containing E. solidaginis larvae.

\subsection{References}

5501 Viecili, P. R. N. et al. in Advances in Clinical Chemistry Vol. 80 (ed Gregory S. Makowski) 1-44 (Elsevier, 2017). Vance, J. E. \& Adeli, K. in Biochemistry of Lipids, Lipoproteins and Membranes (eds E. Vance Dennis \& E. Vance Jean) Ch. 18, 507-531 (Elsevier, 2008). Ståhlman, M. et al. Clinical dyslipidaemia is associated with changes in the lipid composition and inflammatory properties of apolipoprotein-B-containing lipoproteins from women with type 2 diabetes. Diabetologia 55, 1156-1166, doi:10.1007/s00125-0112444-6 (2012).

4 Marten, B., Pfeuffer, M. \& Schrezenmeir, J. Medium-chain triglycerides. International Dairy Journal 16, 1374-1382, doi:https://doi.org/10.1016/i.idairyj.2006.06.015 (2006).

5 Kaur, N., Chugh, V. \& Gupta, A. K. Essential fatty acids as functional components of foodsa review. Journal of food science and technology 51, 2289-2303 (2014).

6 St-Onge, M.-P. \& Jones, P. J. Physiological effects of medium-chain triglycerides: potential agents in the prevention of obesity. The Journal of nutrition 132, 329-332 (2002).

7 Nanji, A. A., Yang, E. K., Fogt, F., Sadrzadeh, S. \& Dannenberg, A. J. Medium chain triglycerides and vitamin $E$ reduce the severity of established experimental alcoholic liver disease. Journal of Pharmacology and Experimental Therapeutics 277, 1694-1700 (1996).

8 Jeppesen, P. \& Mortensen, P. The influence of a preserved colon on the absorption of medium chain fat in patients with small bowel resection. Gut 43, 478-483 (1998).

9 Kim, S.-h. Monoacetyldiacylglycerol Derivative for the Treatment of Sepsis. (2010). 
57010 Hong, J. J. et al. Enteral administration of a synthetic monoacetyldiglyceride improves survival in a murine model of abdominal sepsis. Journal of Trauma and Acute Care Surgery 68, 62-68 (2010).

11 Kim, S.-h. Immunomodulating Agent, Anti-Cancer Agent and Health Food Containing Monoacetyldiacylglycerol Derivatives. (2008).

$12 \mathrm{Kim}, \mathrm{J}$. W. et al. Compositions containing monoacetyldiacylglycerol compound as an active ingredient for preventing or treating rheumatoid arthritis. (2018).

13 Oh, S.-R. et al. Composition containing monoacetyldiacylglycerol compound as active ingredient for preventing or treating asthma. (2018).

14 Durrett, T. P. et al. A distinct DGAT with sn-3 acetyltransferase activity that synthesizes unusual, reduced-viscosity oils in Euonymus and transgenic seeds. Proceedings of the National Academy of Sciences 107, 9464-9469 (2010).

15 Liu, J. et al. Metabolic engineering of oilseed crops to produce high levels of novel acetyl glyceride oils with reduced viscosity, freezing point and calorific value. Plant biotechnology journal 13, 858-865 (2015).

16 Litchfield, C. Analysis of triglycerides. (Elsevier, 2012).

17 Zhou, S. Quantitation of Lipid Classes by Thin-Layer Chromatography with Flame Ionization Detection. Current Protocols in Food Analytical Chemistry 7, D1. 6.1-D1. 6.14 (2003).

18 Xie, D. et al. Antarctic Krill (Euphausia superba) Oil: A Comprehensive Review of Chemical Composition, Extraction Technologies, Health Benefits, and Current Applications. Comprehensive Reviews in Food Science and Food Safety 18, 514-534, doi:10.1111/15414337.12427 (2019).

19 Sinanoglou, V. J. et al. On the combined application of latroscan TLC-FID and GC-FID to identify total, neutral, and polar lipids and their fatty acids extracted from foods. International Scholarly Research Notices 2013 (2013).

20 Marshall, K. E. et al. Seasonal accumulation of acetylated triacylglycerols by a freezetolerant insect. Journal of Experimental Biology 217, 1580-1587 (2014).

21 Ratnayake, W. M. \& Galli, C. Fat and fatty acid terminology, methods of analysis and fat digestion and metabolism: a background review paper. Ann Nutr Metab 55, 8-43, doi:10.1159/000228994 (2009).

22 Narváez-Rivas, M. \& Zhang, Q. Comprehensive untargeted lipidomic analysis using coreshell C30 particle column and high field orbitrap mass spectrometer. Journal of Chromatography A 1440, 123-134, doi:https://doi.org/10.1016/i.chroma.2016.02.054 (2016).

23 Chen, Y. et al. Regioisomeric and enantiomeric analysis of primary triglycerides in human milk by silver ion and chiral HPLC APCI-MS. Journal of Dairy Science (2020).

24 Boyce, G. et al. Using liquid chromatography mass spectrometry (LC-MS) to assess the effect of age, high-fat diet, and rat strain on the liver metabolome. PloS one 15, e0235338 (2020).

25 Folch, J., Lees, M. \& Stanley, G. H. S. A simple method for the isolation and purification of total lipides from animal tissues. Journal of Biological Chemistry 226, 497-509 (1957).

26 Pham, T. H. et al. Targeting modified lipids during routine lipidomics analysis using HILIC and C30 reverse phase liquid chromatography coupled to mass spectrometry. Scientific Reports 9, 1-15 (2019).

27 Vidal, N. P. et al. The use of natural media amendments to produce kale enhanced with functional lipids in controlled environment production system. Scientific Reports 8, 1-14 (2018). 
Lee, J. W., Nagai, T., Gotoh, N., Fukusaki, E. \& Bamba, T. Profiling of regioisomeric triacylglycerols in edible oils by supercritical fluid chromatography/tandem mass spectrometry. J Chromatogr B Analyt Technol Biomed Life Sci 966, 193-199, doi:10.1016/j.jchromb.2014.01.040 (2014).

29 Leskinen, H. M., Suomela, J.-P. \& Kallio, H. P. Quantification of triacylglycerol regioisomers by ultra-high-performance liquid chromatography and ammonia negative ion atmospheric pressure chemical ionization tandem mass spectrometry. Rapid Commun. Mass Spectrom. 24, 1-5 ( 2010).

30 Kallio, H., Nylund, M., Bostrom, P. \& Yang, B. Triacylglycerol regioisomers in human milk resolved with an algorithmic novel electrospray ionization tandem mass spectrometry method. Food Chem 233, 351-360, doi:10.1016/j.foodchem.2017.04.122 (2017).

31 Han, X. Lipidomics: Comprehensive mass spectrometry of lipids. (John Wiley \& Sons, 2016).

32 Pham, T. H. et al. Targeting Modified Lipids during Routine Lipidomics Analysis using HILIC and C30 Reverse Phase Liquid Chromatography coupled to Mass Spectrometry. Sci Rep 9 , 5048, doi:10.1038/s41598-019-41556-9 (2019).

33 Williams, C. M., Thomas, R. H., MacMillan, H. A., Marshall, K. E. \& Sinclair, B. J. Triacylglyceride measurement in small quantities of homogenised insect tissue: comparisons and caveats. Journal of Insect Physiology 57, 1602-1613 (2011).

34 Sinanoglou, V. J. et al. Monitoring the quality of $\gamma$-irradiated macadamia nuts based on lipid profile analysis and Chemometrics. Traceability models of irradiated samples. Food research international 60, 38-47 (2014).

35 Katsoyannos, E. et al. Quality parameters of olive oil from stoned and nonstoned Koroneiki and Megaritiki Greek olive varieties at different maturity levels. Grasas y Aceites 66, 067 (2015).

36 Akanbi, T. O. \& Barrow, C. J. Compositional information useful for authentication of krill oil and the detection of adulterants. Food analytical methods 11, 178-187 (2018).

37 Wang, L., Zhou, X. \& Wan, X. Research progress on unusual acetyl triacylglycerol. Oil Crop Science 2, 211 (2017).

38 Andrikopoulos, N. K. Chromatographic and spectroscopic methods in the analysis of triacylglycerol species and regiospecific isomers of oils and fats. Critical reviews in food science and nutrition 42, 473-505 (2002).

39 Bird, S. S., Marur, V. R., Sniatynski, M. J., Greenberg, H. K. \& Kristal, B. S. Serum lipidomics profiling using LC-MS and high-energy collisional dissociation fragmentation: focus on triglyceride detection and characterization. Analytical chemistry 83, 6648-6657 (2011).

40 Lee, K. W. Y., Porter, C. J. \& Boyd, B. J. A simple quantitative approach for the determination of long and medium chain lipids in bio-relevant matrices by high performance liquid chromatography with refractive index detection. Aaps Pharmscitech 14, 927-934 (2013).

41 Wang, W., Tian, S. \& Stark, R. E. Isolation and identification of triglycerides and ester oligomers from partial degradation of potato suberin. Journal of agricultural and food chemistry 58, 1040-1045 (2010).

42 Ryu, H.-M., Jeong, Y.-S., Yim, C.-S., Lee, J.-H. \& Chung, S.-J. Quantification of EC-18, a synthetic monoacetyldiglyceride (1-palmitoyl-2-linoleoyl-3-acetyl-rac-glycerol), in rat and mouse plasma by liquid-chromatography/tandem mass spectrometry. Journal of pharmaceutical and biomedical analysis 137, 155-162 (2017). 
691

692

693

694

695
43 McAnoy, A. M., Wu, C. C. \& Murphy, R. C. Direct qualitative analysis of triacylglycerols by electrospray mass spectrometry using a linear ion trap. Journal of the American Society for Mass Spectrometry 16, 1498-1509 (2005).

44 Yang, H. O. et al. Purification and structural determination of hematopoietic stem cellstimulating monoacetyldiglycerides from Cervus nippon (deer antler). Chemical and pharmaceutical bulletin 52, 874-878 (2004).

45 Kim, Y. H., Han, S. Y., Cho, S. H., Yoo, J. S. \& Jhon, G. J. Structural determination of synthetic monoacetyldiglycerides by tandem mass spectrometry of sodium-adducted molecules. Rapid communications in mass spectrometry 13, 481-487 (1999).

46 Dolendo, A., Means, J., Tobias, J. \& Perkins, E. Synthesis and Mass Spectral Properties of a Triglyceride Precursor of $\delta$-Dodecalactone. Journal of Dairy Science 52, 21-26 (1969).

47 Gao, X. et al. A reversed-phase capillary ultra-performance liquid chromatography-mass spectrometry (UPLC-MS) method for comprehensive top-down/bottom-up lipid profiling. Analytical and bioanalytical chemistry 402, 2923-2933 (2012).

48 Pham, T. H. et al. Moose and Caribou as Novel Sources of Functional Lipids: Fatty Acid Esters of Hydroxy Fatty Acids, Diglycerides and Monoacetyldiglycerides. Molecules 24, doi:10.3390/molecules24020232 (2019).

49 Liu, J. et al. Field production, purification and analysis of high-oleic acetyl-triacylglycerols from transgenic Camelina sativa. Industrial Crops and Products 65, 259-268, doi:https://doi.org/10.1016/j.indcrop.2014.11.019 (2015).

50 Van Huis, A. Potential of insects as food and feed in assuring food security. Annual review of entomology 58, 563-583 (2013).

51 Dobermann, D., Swift, J. \& Field, L. Opportunities and hurdles of edible insects for food and feed. Nutrition Bulletin 42, 293-308 (2017).

52 Lee, J. W., Nagai, T., Gotoh, N., Fukusaki, E. \& Bamba, T. Profiling of regioisomeric triacylglycerols in edible oils by supercritical fluid chromatography/tandem mass spectrometry. Journal of Chromatography B 966, 193-199 (2014). 

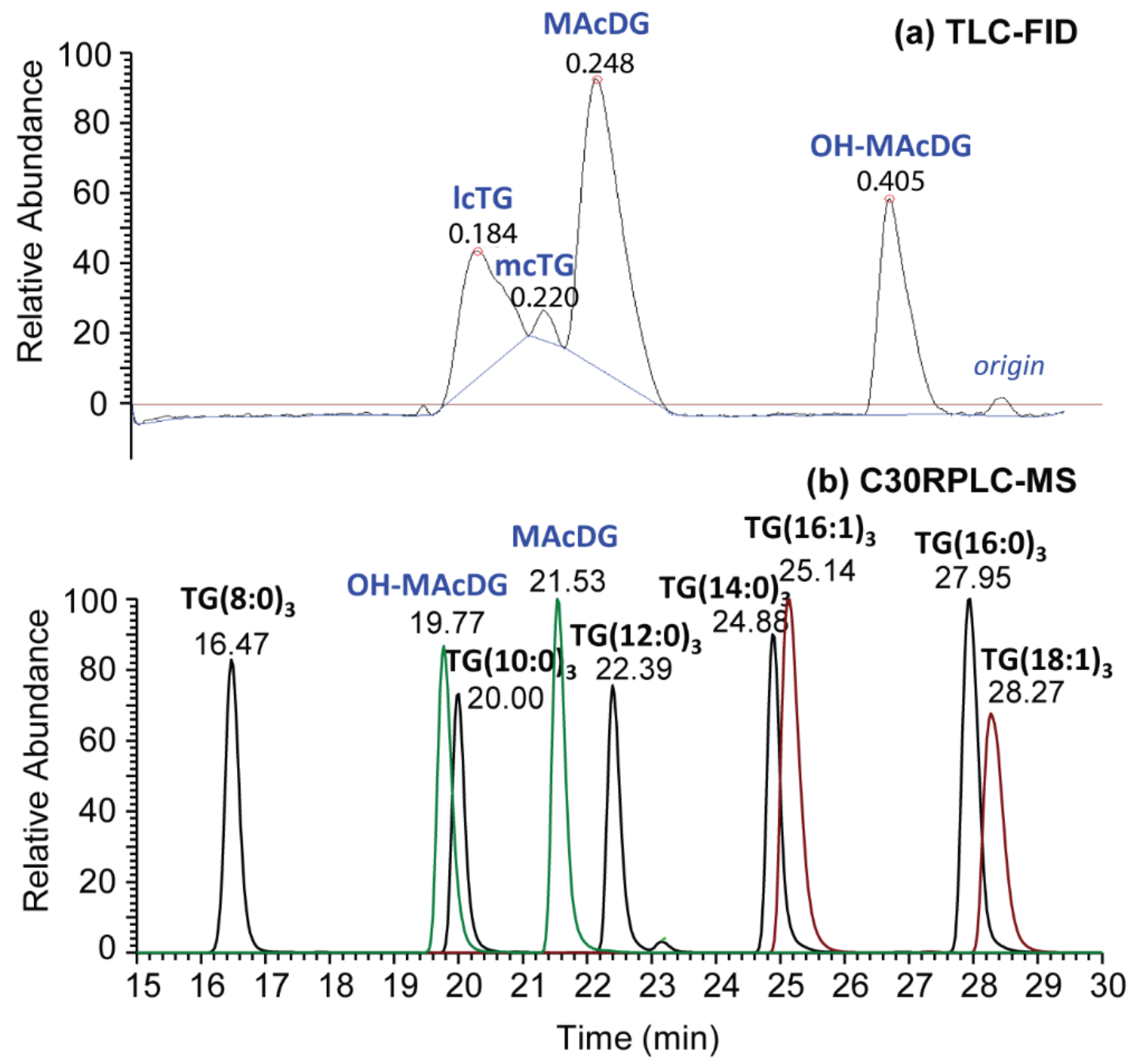

700

701

702

703

704

705
Fig. 1 a) TLC-FID separation of TG subclasses and b) C30-RPLC-HESI-HRAM-MS/MS chromatograms in positive ion mode of the standard lipid mixture of monoacetyldiacylglycerols (MACDG), hydroxylated monoacetyldiacylglycerols (OH-MAcDG) medium and long chain triglycerides (mcTG and IcTG). 

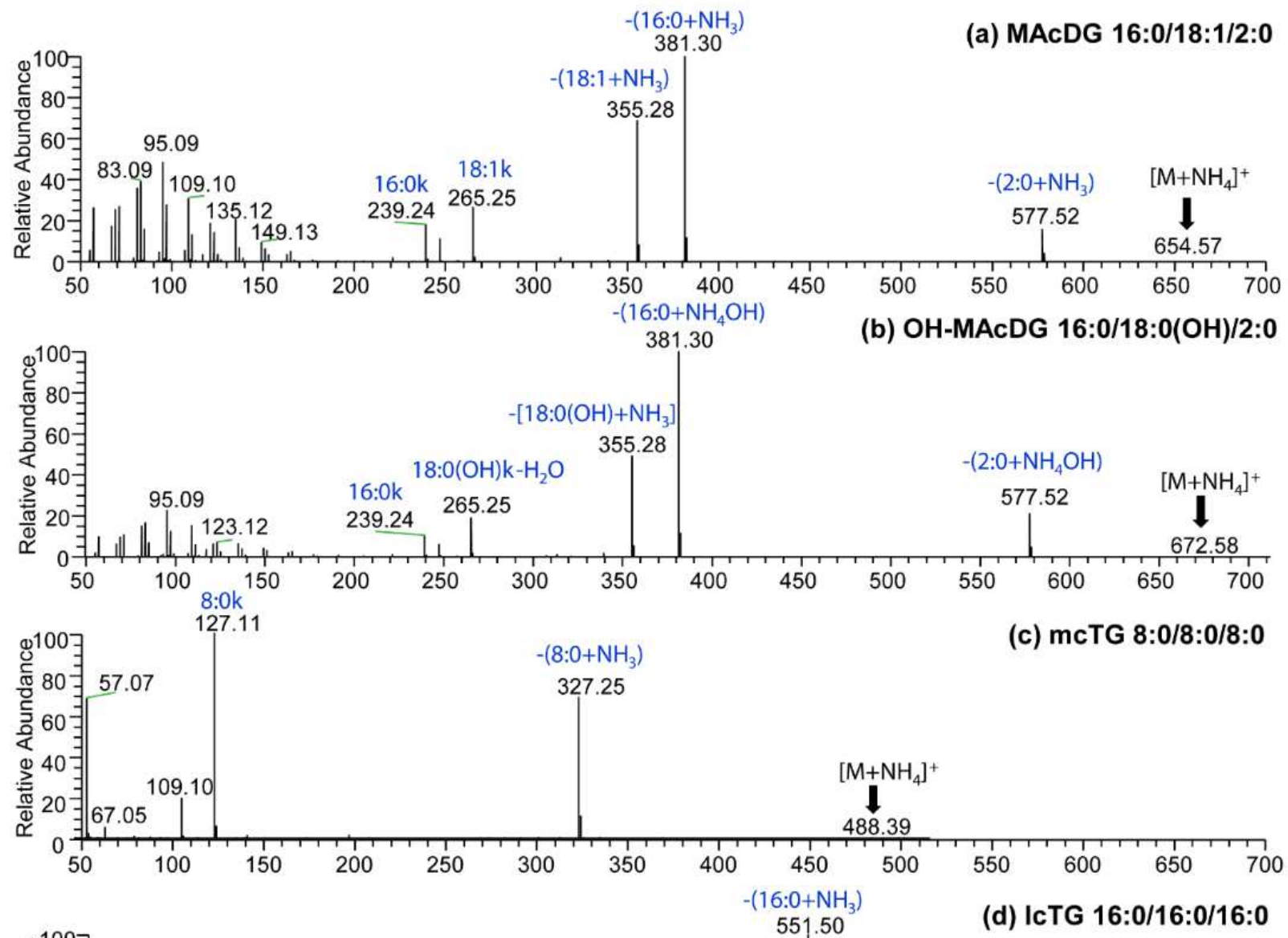

706

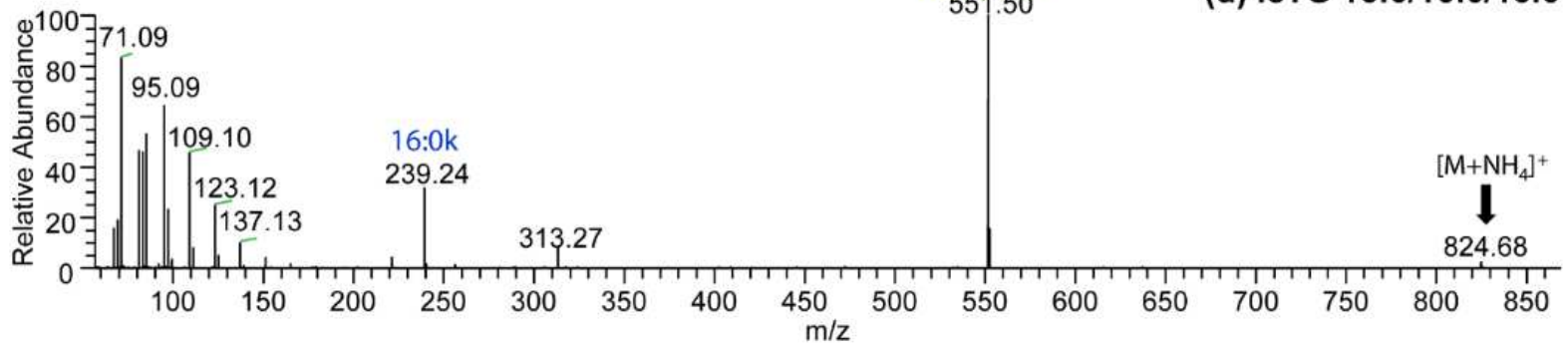

707 Fig. 2 C30-RPLC-HESI-HRAM-MS/MS spectra showing fragmentation of precursor ions isolated at

7081 Da selection window in positive ion mode for the lipid standards representing: a) MAcDG

$70916: 0 / 18: 1 / 2: 0(36: 1)$ at $m / z$ 654.57, b) OH-MAcDG 16:0/18:0(OH)/2:0 (OH-36:0) at $\mathrm{m} / z$ 672.58, c)

710 mcTG 8:0/8:0/8:0 (24:0) at $\mathrm{m} / z \quad 488.39$ and, d) IcTG 16:0/16:0/16:0 at $\mathrm{m} / \mathrm{z}$ 824.68. All TG

711 subclasses are shown as ammonium adducts $\left(\left[\mathrm{M}+\mathrm{NH}_{4}\right]^{+}\right.$.

712

713 

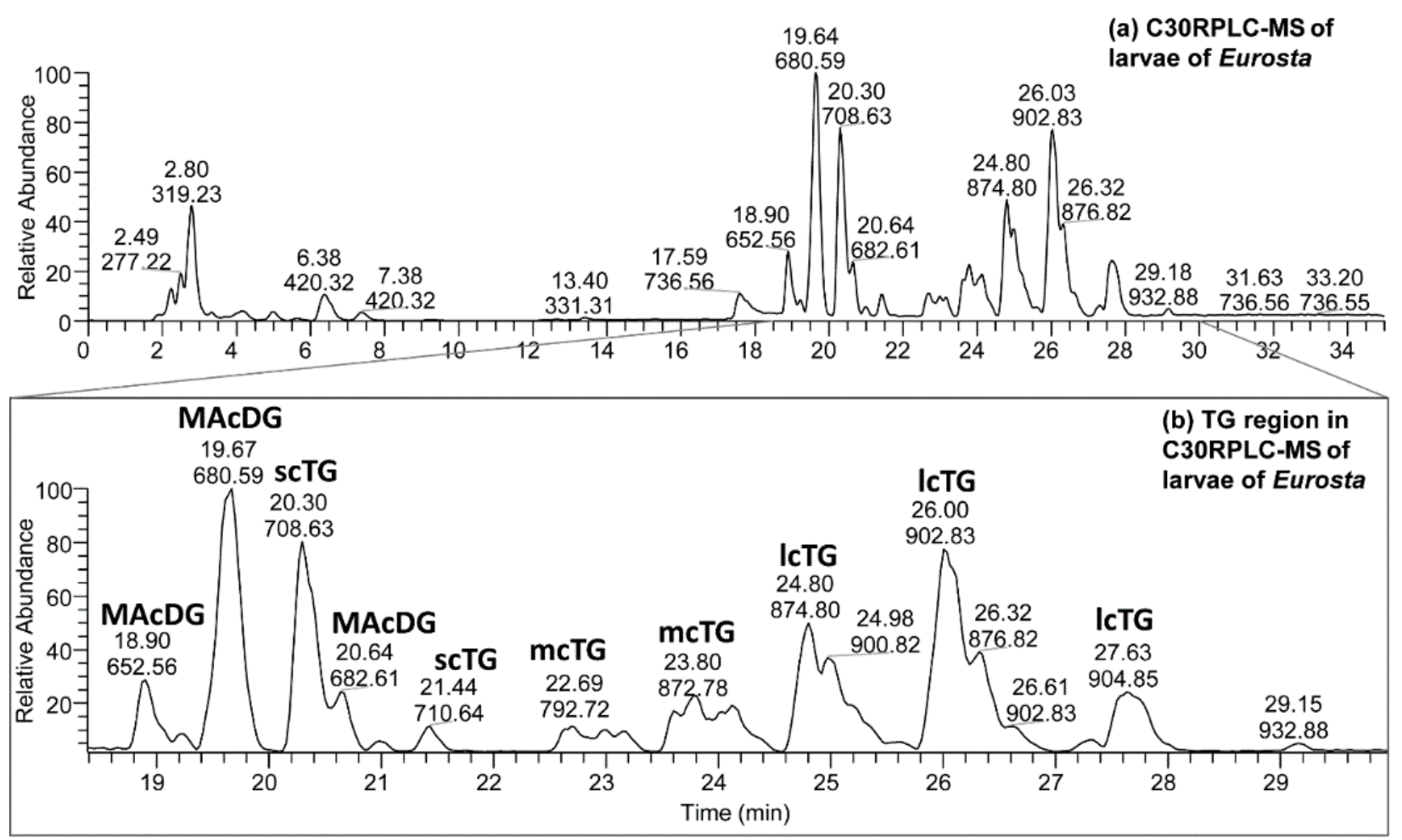

715 Fig. 3 a) C30-RPLC-HESI-HRAM-MS/MS chromatogram in positive ion mode of complex lipid 716 extracted from the larvae of Eurosta solidaginis species. b) Zoomed in neutral lipids region of the 717 C30-RPLC-MS chromatogram showing some major MAcDG, scTG, mcTG and IcTG contribution of 718 the lipid profile. 

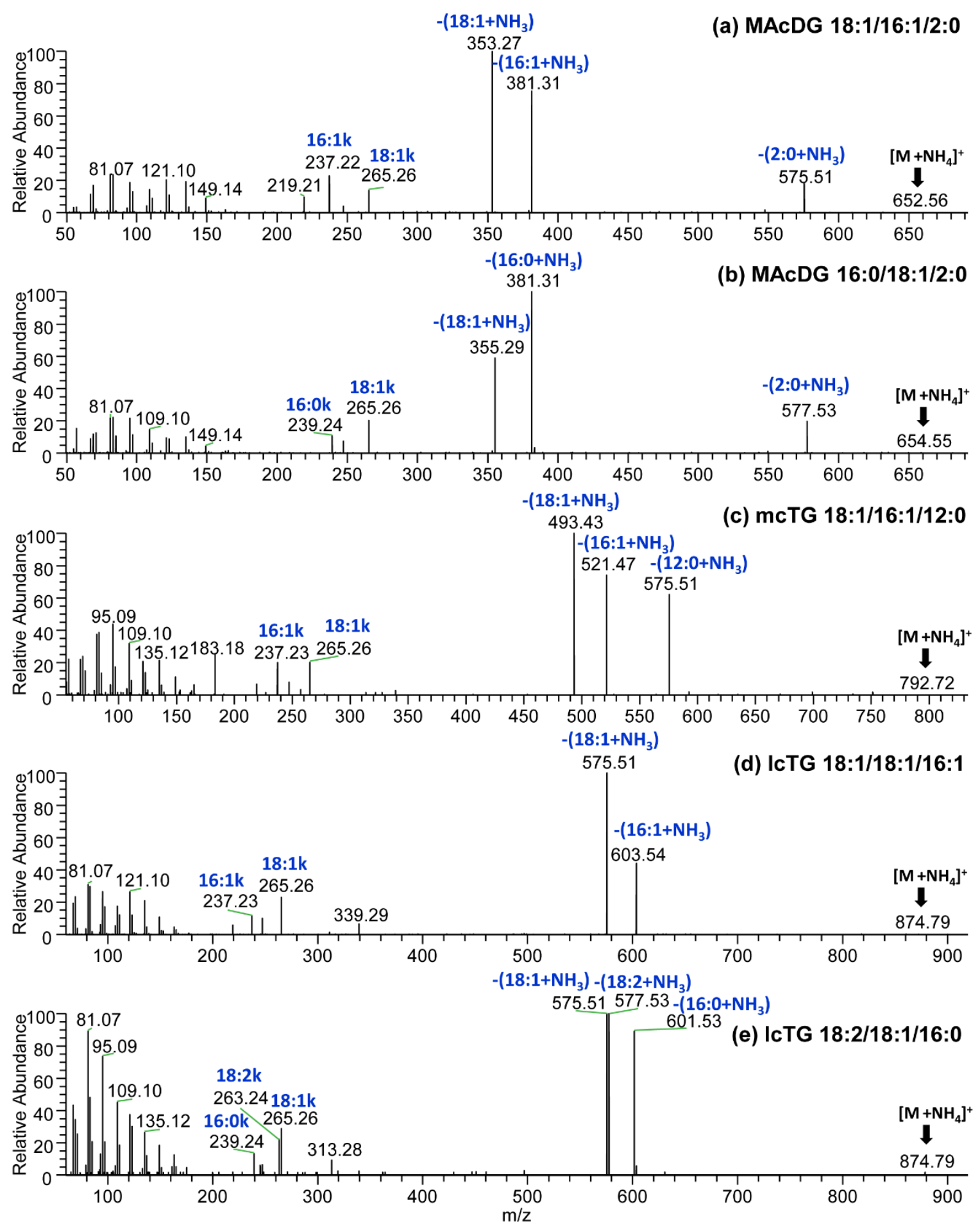

722 Fig. 4 C30-RPLC-HESI-HRAM-MS/MS spectra showing fragmentation of precursor ions isolated at 7231 Da selection window in positive ion mode for the major MAcDG species in Eurosta solidaginis 724 species larvae corresponding to: a) MAcDG 18:1/16:1/2:0 (36:2) at $\mathrm{m} / \mathrm{z}$ 652.56, b) MAcDG $72516: 0 / 18: 1 / 2: 0$ (36:1) at $m / z$ 654.55. c) mcTG 18:1/16:1/12:0 (38:3) $m / z$ 678.56. Resolution of IcTG $72652: 3$ isomers at $m / z$ 874.79 representing: d) $18: 1 / 18: 1 / 16: 1$ and e $18: 2 / 18: 1 / 16: 0$. 


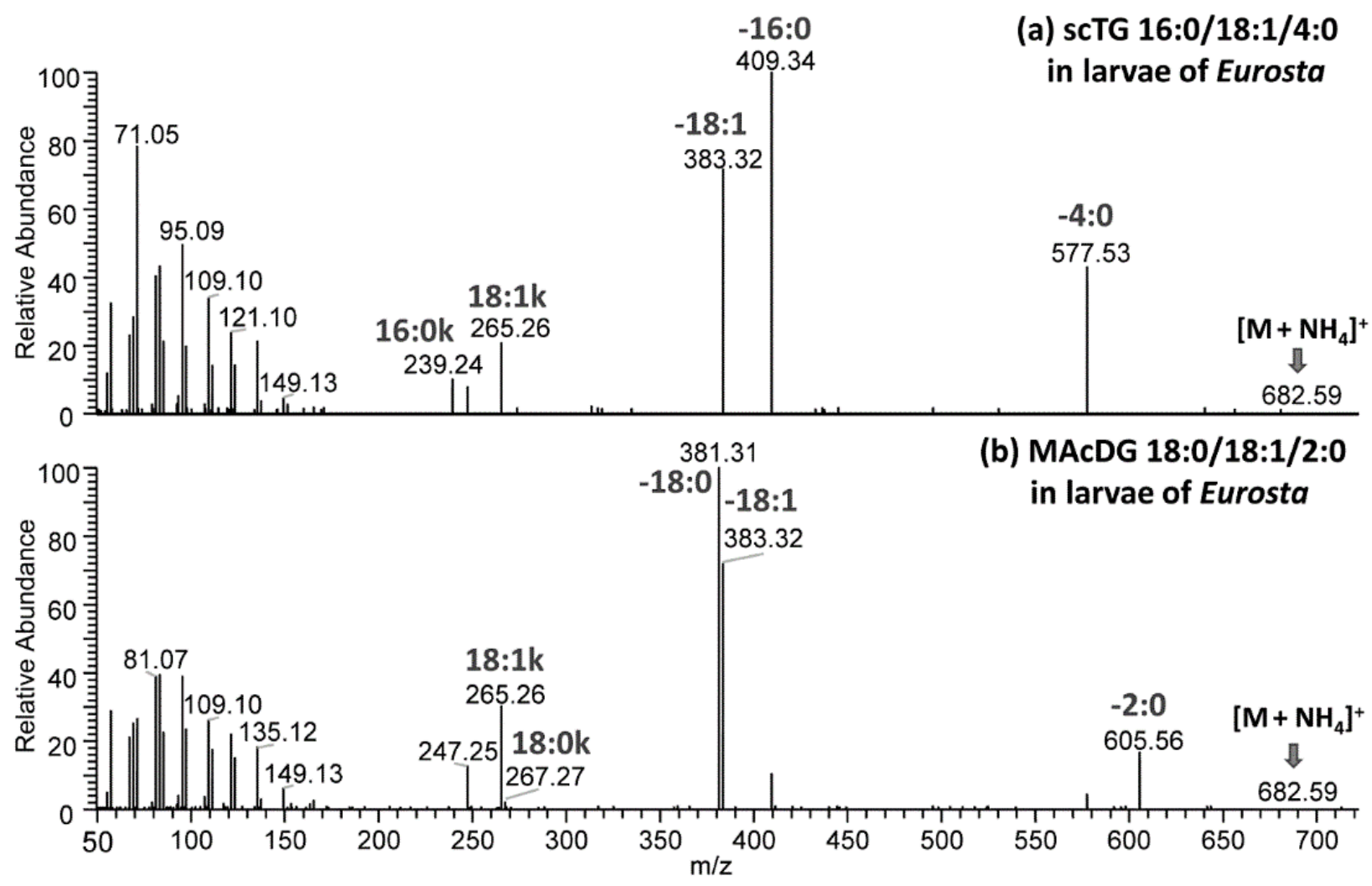

729 Fig. 5 C30-RPLC-HESI-HRAM-MS/MS spectra showing fragmentation of precursor ions ions 730 isolated at 1 Da selection window in positive ion mode for isomers of scTG and MAcDG in Eurosta 731 solidaginis corresponding to: a) scTG 16:0/18:1/4:0 (38:1) at $\mathrm{m} / \mathrm{z} 682.59$ and b) MAcDG $732 \quad 18: 0 / 18: 1 / 2: 0(38: 1)$ at $m / z 682.59$. 
(a)

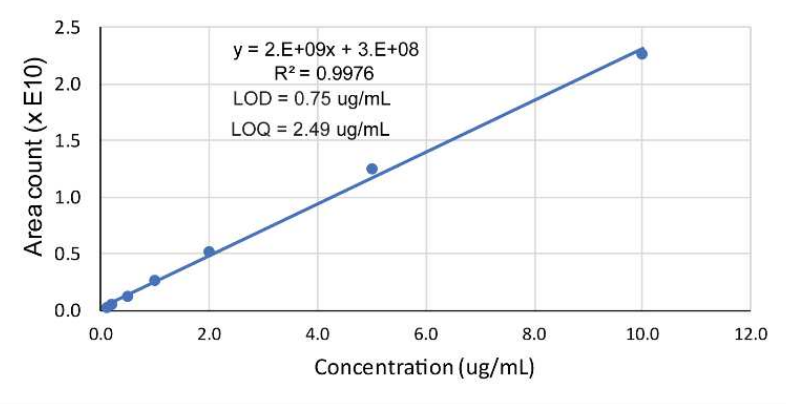

(b)

$\operatorname{MACDG}(16: 0 / 18: 2 / 2: 0)+\mathrm{NH}_{4}^{+}$

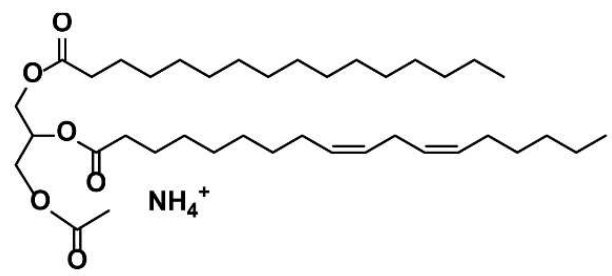

(c) MAcDG subclass percentage composition of E. solidiginis

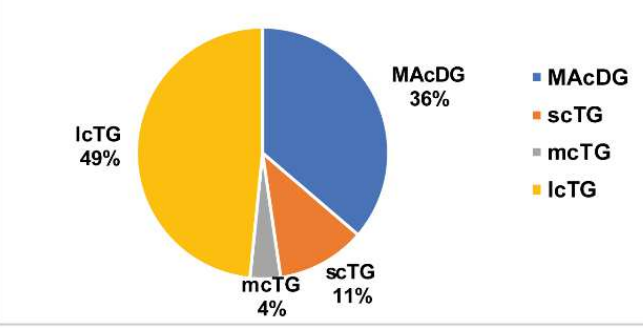

(d) MAcDG subclass content in larvae of E. solidaginis

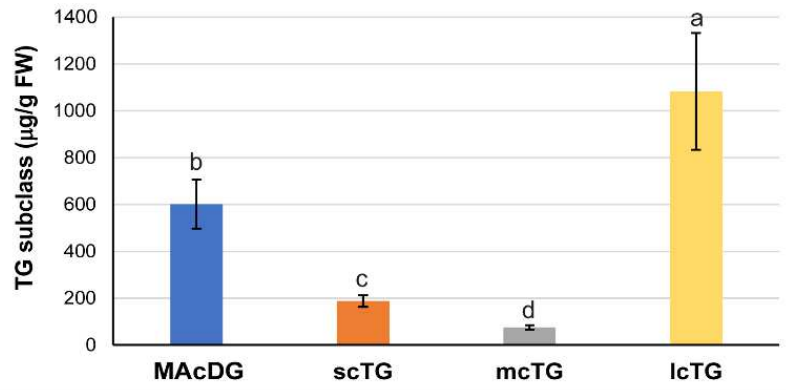

(e)

MACDG molecular distribution in the larvae of $E$. solidaginis
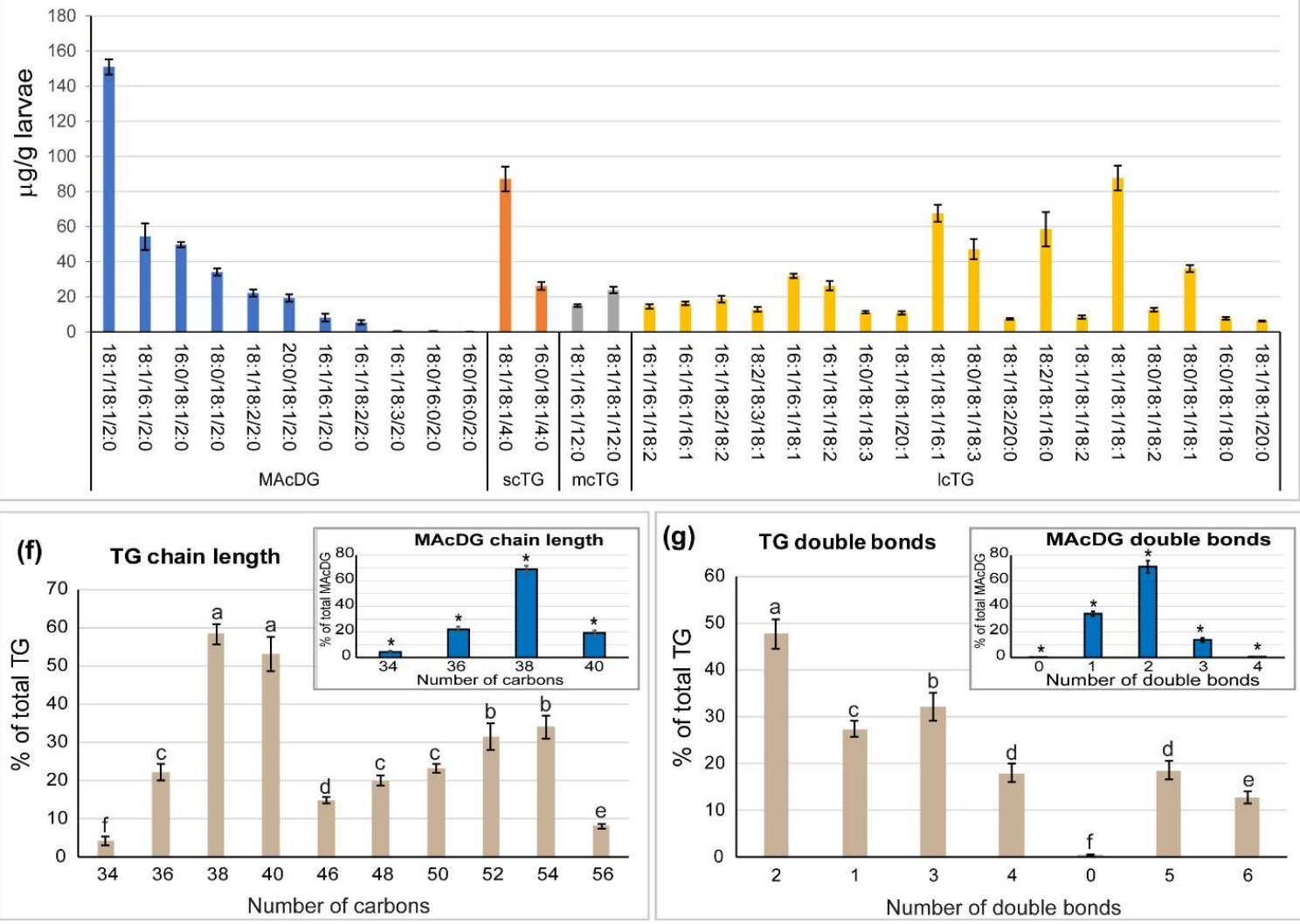
737 Fig. 6 Quantitation and distribution of $C$ and other TG subclasses present in the larvae of $E$.

738 solidaginis. a) Standard curve used to calculate the amount of MAcDG in E. solidaginis, b)

739 Representative structure of MAcDG, c) Percent composition, d) quantity, and e) molecular species

740 distribution of MACDG and other TG subclasses present in the larvae of E. solidaginis. f) Chain

741 length as the number of carbons, $\mathrm{g}$ ) and number of double bonds in the sum of the FA moieties

742 in MAcDG present in E. solidaginis. Values represent means \pm standard error $(N=7)$. Fisher LSD

743 was used to separate the means at alpha $=0.05$ following analysis using one-way ANOVA. 


\section{Supplementary Files}

This is a list of supplementary files associated with this preprint. Click to download.

- GraphicalAbstract.docx

- ManfuletalSupplementaryInformationMAcDGOct28.docx 